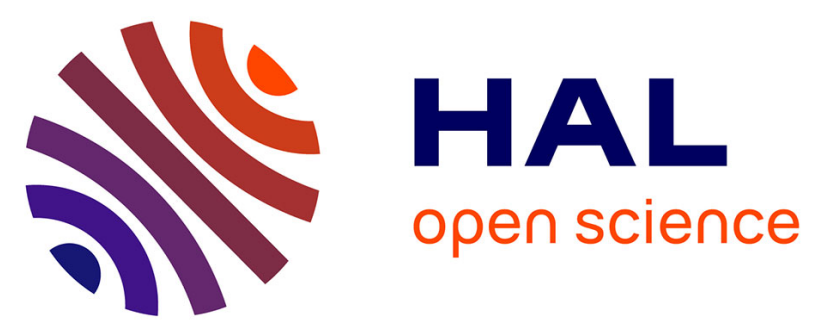

\title{
Effective Coefficients and Local Fields of Periodic Fibrous Piezocomposites with 622 Hexagonal Constituents
}

\author{
Ransés Alfonso-Rodríguez, Julian Bravo-Castillero, Renald Brenner, Raúl \\ Guinovart-Díaz, Leslie D Pérez-Fernández, Reinaldo Rodríguez-Ramos, \\ Federico J Sabina
}

\section{To cite this version:}

Ransés Alfonso-Rodríguez, Julian Bravo-Castillero, Renald Brenner, Raúl Guinovart-Díaz, Leslie D Pérez-Fernández, et al.. Effective Coefficients and Local Fields of Periodic Fibrous Piezocomposites with 622 Hexagonal Constituents. Generalized Models and Non-Classical Approaches in Complex Materials, In press. hal-01727347

\section{HAL Id: hal-01727347 https://hal.sorbonne-universite.fr/hal-01727347}

Submitted on 9 Mar 2018

HAL is a multi-disciplinary open access archive for the deposit and dissemination of scientific research documents, whether they are published or not. The documents may come from teaching and research institutions in France or abroad, or from public or private research centers.
L'archive ouverte pluridisciplinaire $\mathbf{H A L}$, est destinée au dépôt et à la diffusion de documents scientifiques de niveau recherche, publiés ou non, émanant des établissements d'enseignement et de recherche français ou étrangers, des laboratoires publics ou privés. 


\title{
Chapter 1 \\ Effective Coefficients and Local Fields of Periodic Fibrous Piezocomposites with 622 Hexagonal Constituents
}

\author{
Ransés Alfonso-Rodríguez, Julián Bravo-Castillero, Renald Brenner, Raúl \\ Guinovart-Díaz, Leslie D. Pérez-Fernández, Reinaldo Rodríguez-Ramos, and \\ Federico J. Sabina
}

\begin{abstract}
The asymptotic homogenization method is applied to a family of boundary value problems for linear piezoelectric heterogeneous media with periodic and rapidly oscillating coefficients. We consider a two-phase fibrous composite consisting of identical circular cylinders perfectly bonded in a matrix. Both constituents are piezoelectric 622 hexagonal crystal and the periodic distribution of the fibers follows a
\end{abstract}

Ransés Alfonso-Rodríguez

Department of Mathematics, University of Central Florida, 4393 Andromeda Loop North, Orlando, FL 32816, USA

e-mail: ranses.alfonsodknights.ucf.edu

Julián Bravo-Castillero

Facultad de Matemática y Computación, Universidad de La Habana, San Lázaro y L, Vedado, CP 10400, La Habana, Cuba \& Instituto de Investigaciones en Matemáticas Aplicadas y en Sistemas, Universidad Nacional Autónoma de México, Delegación Álvaro Obregón, Apartado Postal 20-126, 01000 CDMX, México

e-mail: jbravo@matcom.uh.cu, julian@mym.iimas.unam.mx

Raúl Guinovart-Díaz · Reinaldo Rodríguez-Ramos

Facultad de Matemática y Computación, Universidad de La Habana, San Lázaro y L, Vedado, CP 10400, La Habana, Cuba

e-mail: jbravo@matcom.uh.cu, guino@matcom.uh.cu, reinaldo@matcom.uh.cu

Renald Brenner

Sorbonne Université, Centre National de la Recherche Scientifique, UMR 7190, Institut Jean Le Rond d'Alembert, F-75005 Paris, France

e-mail: renald.brennereupmc.fr

Leslie D. Pérez-Fernández

Universidade Federal de Pelotas, Departamento de Matemática e Estatística, Instituto de Física e Matemática, Caixa Postal 354, CEP 96010-900, Pelotas, Rio Grande do Sul, Brazil

e-mail: leslie.fernandez@ufpel.edu.br

Federico J. Sabina

Instituto de Investigaciones en Matemáticas Aplicadas y en Sistemas, Universidad Nacional Autónoma de México, Delegación Álvaro Obregón, Apartado Postal 20-126, 01000 CDMX, México e-mail: fjs@mym. iimas.unam.mx 
rectangular array. Closed-form expressions are obtained for the effective coefficients, based on the solution of local problems using potential methods of a complex variable. An analytical procedure to study the spatial heterogeneity of the strain and electric fields is described. Analytical expressions for the computation of these fields are given for specific local problems. Examples are presented for fiber-reinforced and porous matrix including comparisons with fast Fourier transform (FFT) numerical results.

\subsection{Introduction}

At the beginning of the nineteen nineties, different homogenization techniques were applied to investigate the macroscopic or effective properties of periodic piezoelectric composites (Galka et al, 1992; Maugin and Turbé, 1991; Telega, 1991; Turbé and Maugin, (1991). The initial studies of the effective dynamical properties of periodic piezoelectric composites by considering Bloch expansions were reported in Telega (1991); Turbé and Maugin (1991). The method of $\Gamma$-convergence was used to study the static effective properties without dispersive behavior. In Galka et al (1992), the two-scale asymptotic homogenization was applied for thermo-piezoelectric heterogeneous media.

In this framework, the computation of the effective properties depends on the solution of the so-called local problems. Many works have been devoted to the application of analytical and numerical techniques for solving the local problems, see, for instance, Berger et al (2003, 2006); Bravo-Castillero et al (1997, 1998, 2001); Galka et al (1996); Otero et al (2003); Rodríguez-Ramos et al (1996); Sabina et al (2001). In general, those efforts have been addressed to piezoelectric composites whose constituents exhibit a $6 \mathrm{~mm}$ symmetry class which are of interest in smart materials applications.

The purpose of this work is essentially twofold. Firstly, to provide closed-form expressions for the effective coefficients of fibrous composites with piezoelectric components which belong to the 622 hexagonal symmetry (Nye, 1957) and with a rectangular distribution of the fibers. These results generalize those published in López-López et al (2005); Aguiar et al (2013) where the periodic cell is a square. Secondly, to describe a procedure to obtain analytical expressions for the components of both the local strain tensor and local electric field intensity vector.

These studies could be interesting for the modeling of biomaterials in bone mechanics applications (for instance, collagen is a natural substance which possesses the 622 symmetry, see Fukada, 1984). In Telega (1991), for the first time, the application of homogenization methods for finding the effective piezoelectric properties of compact bones was sketched. However, up to now, few papers on composites with 622 symmetry have been reported (Aguiar et al, 2013; Alfonso-Rodríguez et al, 2017, López-López et al, 2005, Sevostianov et al, 2014).

The paper is organized as follows. In Sect. 1.2, a family of boundary value problems for periodic piezoelectric media with rapidly oscillating coefficients is presented

Page:2 job:editor macro:svmult.cls date/time:25-Jan-2018/8:32 
in matrix notation. The main steps of the asymptotic homogenization procedure to obtain the averaged problem, the local problems, the effective coefficients and the components of the local fields, are summarized. In Sect. 1.4, the homogenization model is applied to the case of unidirectional fibrous composites with 622 piezoelectric phases and a rectangular periodic cell. The relevant local problems are solved based on the theory of functions of a complex variable and closed-form expressions are derived for the corresponding effective coefficients. Analytical expressions are also explicitly given for the components of the strain and electric local fields associated with particular local problems. In Sect. 1.6, some numerical examples are presented and the accuracy of the results is assessed through comparisons with results derived from the FFT numerical scheme (Brenner, 2009, 2010).

\subsection{A Boundary Value Problem of the Linear Piezoelectricity Theory}

Let $\Omega \subset \mathbb{R}^{3}$ be a three-dimensional domain with infinitely smooth boundary $\partial \Omega$. The material properties of a piezoelectric body occupying $\Omega$ are described by elastic $\left(c_{i j k l}\right)$, piezoelectric $\left(e_{i j k}\right)$, and dielectric $\left(\kappa_{i j}\right)$ coefficients. These coefficients are assumed to be differentiable, rapidly oscillating and $\varepsilon Y$-periodic functions in the local variable $y=x / \varepsilon$, where $x=\left(x_{1}, x_{2}, x_{3}\right) \in \Omega$ is the global variable, $\varepsilon>0$ is the usual small geometric parameter, and $Y$ is the periodic cell.

The material functions are defined by

$c_{i j k l}^{\varepsilon}(x)=c_{i j k l}\left(\frac{x}{\varepsilon}\right), \quad e_{i j k}^{\varepsilon}(x)=e_{i j k}\left(\frac{x}{\varepsilon}\right), \quad \kappa_{i j}^{\varepsilon}(x)=\kappa_{i j}\left(\frac{x}{\varepsilon}\right), \quad i, j, k=1,2,3$,

which are denoted in a unified fashion by $A_{j l} \equiv\left(a_{j l}^{i^{\prime} k^{\prime}}\right)_{i^{\prime}, k^{\prime}=1, \ldots, 4}$, where

$$
a_{j l}^{i k}=c_{i j k l,} \quad a_{j l}^{i 4}=e_{l i j,} \quad a_{j l}^{4 k}=e_{j k l,} \quad a_{j l}^{44}=-\kappa_{j l} .
$$

The material functions satisfy the usual symmetry conditions

$$
a_{j l}^{i k}=a_{i l}^{j k}=a_{j k}^{i l}=a_{l j}^{k i}, \quad a_{j l}^{i 4}=a_{i l}^{j 4}, \quad a_{j l}^{4 k}=a_{j k}^{4 l}, \quad a_{j l}^{44}=a_{l j}^{44},
$$

and we will assume that there exist a constant $\varkappa>0$ such that, for any symmetric matrix $q=\left(q_{i j}\right)$ and any vector $a=\left(a_{i}\right)$

$$
a_{j l}^{i k}(x) q_{i j} q_{k l} \geq \varkappa q_{i j} q_{i j}, \quad a_{j l}^{44}(x) a_{j} a_{l} \geq \varkappa a_{j} a_{j} .
$$

Note that the summation rule on the repeated indices will be used throughout the paper.

A boundary value problem for the system of equations of linear piezoelectricity can be written as

Page: 3 job:editor macro:svmult.cls date/time:25-Jan-2018/8:32 


$$
\begin{aligned}
\frac{\partial}{\partial x_{j}}\left(A_{j l}^{\varepsilon}(x) \frac{\partial}{\partial x_{l}} U^{\varepsilon}(x)\right) & =0 \in \Omega, \\
U^{\varepsilon}(x) & =\widehat{U}(x), \quad x \in \partial \Omega,
\end{aligned}
$$

where $U^{\varepsilon}(x)=\left(u_{1}^{\varepsilon}(x), u_{2}^{\varepsilon}(x), u_{3}^{\varepsilon}(x), v^{\varepsilon}(x)\right)^{T}$ and $\widehat{U}(x)=\left(\hat{u}_{1}(x), \hat{u}_{2}(x), \hat{u}_{3}(x), \hat{v}(x)\right)^{T}$ represent the unknown and the prescribed boundary conditions, respectively. The superscript $T$ stands for transposition. Equation (1.3) represent a system of partial differential equations to find the mechanical displacement field $u^{\varepsilon}=\left(u_{k}^{\varepsilon}\right)$ and the electric potential $v^{\varepsilon}$. The problem (1.3)-(1.4) describes the piezoelectric state of a composite material that occupies the domain $\Omega$ and is free of external forces.

\subsection{Homogenization, Local Problems and Effective Coefficients}

In this section, the asymptotic homogenization method (AHM) (Bakhvalov and Panasenko, 1989) is applied to the family of problems (1.3)-(1.4). More specifically, the methodology used in Sixto-Camacho et al (2013) is followed.

The solution of (1.3)-(1.4) is sought in the form

$$
U^{\varepsilon}(x)=U^{(0)}(x, y)+\varepsilon U^{(1)}(x, y)+\cdots+\varepsilon^{i} U^{(i)}(x, y)+\ldots,
$$

where

$$
U^{(i)}=\left(u_{1}^{(i)}, u_{2}^{(i)}, u_{3}^{(i)}, v^{(i)}\right)^{T}, \quad i=0,1,2, \ldots
$$

being $u_{k}^{(0)}(x, y), u_{k}^{(1)}(x, y), \ldots, v^{(0)}(x, y), v^{(1)}(x, y), \ldots$ infinitely differentiable and $Y$-periodic functions with respect to $y$. Substituting (1.5) into (1.3)-(1.4), applying the differentiation chain rule and equating to zero the terms corresponding to equal powers of $\varepsilon$ (from $\varepsilon^{-2}, \varepsilon^{-1}, \varepsilon^{0}, \ldots$ ), a recurrent family of partial differential equations is obtained. From the term corresponding to $\varepsilon^{-2}$, it is possible to conclude that the non-perturbed terms of the asymptotic (1.5) are independent of $y$, that is $U^{(0)}=U^{(0)}(x)$. From the term that corresponds to $\varepsilon^{-1}$ the local problems are obtained, which have a solution $U^{(1)}(x, y)$ in the class of $Y$-periodic functions with respect to $y$. Such a solution can be expressed using the method of separable variables as follows

$$
U^{(1)}(x, y)=N^{p}(y) \frac{\partial U^{(0)}(x)}{\partial x_{p}},
$$

with

$$
N^{p}(y)=\left(\begin{array}{cc}
\Xi_{k}^{p q}(y) & \Upsilon_{k}^{p}(y) \\
\Theta^{p q}(y) & \Pi^{p}(y)
\end{array}\right)_{k, q=1,2,3},
$$

where the matrix $N^{p}(y)$ is a $Y$-periodic solution of

$$
\frac{\partial}{\partial y_{j}}\left(A_{j p}(y)+A_{j l}(y) \frac{\partial N^{p}(y)}{\partial y_{l}}\right)=0 .
$$


Based on the periodicity and ellipticity of the material coefficients it is possible to apply the theorem of the Appendix of Sixto-Camacho et al (2013) to prove that equations (1.7) have a unique $Y$-periodic solution up to an additive constant. The problems involving such equations are the so-called local problems. The solutions of such problems play an important role for the calculation of the effective coefficients. Usually, the condition of null average of the local functions (i.e., $\left\langle N^{p}(y)\right\rangle=0$ ) on the periodic cell is imposed for uniqueness. The angular brackets denote the average per unit volume over the cell i.e.

$$
\langle g(y)\rangle=\frac{1}{|Y|} \int_{Y} g(y) d y .
$$

On the other hand, from the terms corresponding to $\varepsilon^{0}$, we obtain the homogenized problem

$$
\begin{aligned}
\underline{A}_{p q} \frac{\partial^{2} U^{(0)}(x)}{\partial x_{p} \partial x_{q}} & =0, \quad x \in \Omega, \\
U^{(0)}(x) & =\widehat{U}(x), \quad x \in \partial \Omega .
\end{aligned}
$$

and the effective coefficients $\underline{A}_{p q}$, which are defined by

$$
\underline{A}_{p q}=\left\langle A_{p q}(y)+A_{p l}(y) \frac{\partial N^{p}(y)}{\partial y_{l}}\right\rangle .
$$

The terms $U^{(i)}(x, y)(i>1)$ of 1.5 can be also expressed in separable variables by

$$
U^{(i)}(x, y)=N^{p p_{1} \ldots p_{i-1}}(y) \frac{\partial^{i} U^{(0)}(x)}{\partial x_{p} \partial x_{p_{1}} \ldots \partial x_{p_{i-1}}},
$$

where $N^{p p_{1} \ldots p_{i-1}}$ are $Y$-periodic solutions of certain partial differential equations which can be found in Eq. (4.13) of Sixto-Camacho et al (2013).

\subsubsection{Explicit Form of the Homogenized Problem, Effective Coefficients and Local Problems}

From (1.8)-1.10, it is possible to obtain the explicit form of the homogenized problem

$$
\begin{gathered}
\underline{c}_{i j k l} \frac{\partial^{2} u_{k}^{(0)}(x)}{\partial x_{j} \partial x_{l}}+\underline{e}_{m i j} \frac{\partial^{2} v^{(0)}(x)}{\partial x_{j} \partial x_{m}}=0, \quad x \in \Omega, \\
\underline{e}_{i k l} \frac{\partial^{2} u_{k}^{(0)}(x)}{\partial x_{i} \partial x_{l}}-\underline{\kappa}_{i m} \frac{\partial^{2} v^{(0)}(x)}{\partial x_{i} \partial x_{m}}=0, \quad x \in \Omega,
\end{gathered}
$$




$$
u_{k}^{(0)}(x)=\hat{u}_{k}(x), \quad v^{(0)}(x)=\hat{v}(x), \quad x \in \partial \Omega
$$

and the effective coefficients

$$
\begin{aligned}
\underline{c}_{i j p q} & =\left\langle c_{i j k l}\left[\delta_{k p} \delta_{l q}+\epsilon_{k l, y}\left(\Xi^{p q}\right)\right]+e_{l i j} \frac{\partial \Theta^{p q}}{\partial y_{l}}\right\rangle, \\
\underline{e}_{j p q} & =\left\langle e_{j k l}\left[\delta_{k p} \delta_{l q}+\epsilon_{k l, y}\left(\Xi^{p q}\right)\right]-\kappa_{j l} \frac{\partial \Theta^{p q}}{\partial y_{l}}\right\rangle, \\
\underline{e}_{p i j} & =\left\langle e_{l i j}\left[\delta_{l p}+\frac{\partial \Pi^{p}}{\partial y_{l}}\right]+c_{i j k l} \epsilon_{k l, y}\left(\Upsilon^{p}\right)\right\rangle, \\
\underline{\kappa}_{i p} & =\left\langle\kappa_{i l}\left[\delta_{l p}+\frac{\partial \Pi^{p}}{\partial y_{l}}\right]-e_{i k l} \epsilon_{k l, y}\left(\Upsilon^{p}\right)\right\rangle,
\end{aligned}
$$

where $\delta_{k l}$ is the Kronecker's delta and

$$
\epsilon_{k l, \xi}(u)=\frac{1}{2}\left(\frac{\partial u_{k}}{\partial \xi_{l}}+\frac{\partial u_{l}}{\partial \xi_{k}}\right)
$$

The local functions $\Xi_{k}^{p q}, \Theta^{p q}, \Upsilon_{k}^{p}$ and $\Pi^{p}$ are $Y$-periodic solutions of the following problems on the cell $Y$ :

- Problem $L^{p q}$ : Find the $Y$-periodic functions $\Xi_{k}^{p q}, \Theta^{p q}$ such that:

$$
\begin{cases}\frac{\partial}{\partial y_{j}}\left\{c_{i j k l}\left[\delta_{k p} \delta_{l q}+\epsilon_{k l, y}\left(\Xi^{p q}\right)\right]+e_{l i j} \frac{\partial \Theta^{p q}}{\partial y_{l}}\right\}=0, & \text { in } Y, \\ \frac{\partial}{\partial y_{j}}\left\{e_{j k l}\left[\delta_{k p} \delta_{l q}+\epsilon_{k l, y}\left(\Xi^{p q}\right)\right]-\kappa_{j l} \frac{\partial \Theta^{p q}}{\partial y_{l}}\right\}=0, & \text { in } Y .\end{cases}
$$

- Problem $L^{p}$ : Find the $Y$-periodic functions $\Upsilon_{k}^{p}, \Pi^{p}$ such that:

$$
\begin{cases}\frac{\partial}{\partial y_{j}}\left\{e_{l i j}\left[\delta_{l p}+\frac{\partial \Pi^{p}}{\partial y_{l}}\right]+c_{i j k l} \epsilon_{k l, y}\left(\Upsilon^{p}\right)\right\}=0, & \text { in } Y, \\ \frac{\partial}{\partial y_{j}}\left\{\kappa_{j l}\left[\delta_{l p}+\frac{\partial \Pi^{p}}{\partial y_{l}}\right]-e_{j k l} \epsilon_{k l, y}\left(\Upsilon^{p}\right)\right\}=0, & \text { in } Y .\end{cases}
$$

\subsubsection{Local Fields}

Now boundary conditions (1.14) are given by linear functions of the type

$$
\hat{u}_{k}(x)=\hat{\varepsilon}_{k l} x_{l}, \quad \hat{v}(x)=-\hat{E}_{l} x_{l},
$$

where $\hat{\varepsilon}_{k l}$ and $\hat{E}_{l}$ are the components of a constant strain tensor and a constant electric field intensity vector, respectively on the boundary of the composite. Under these 
conditions, the functions $u_{k}^{(0)}(x)=\hat{\varepsilon}_{k l} x_{l}$ and $v^{(0)}(x)=-\hat{E}_{l} x_{l}$ represent the solution of the homogenized problem (1.12)-(1.14). So the linearity of $U^{(0)}(x)$ implies that $U^{(i)}(x, y)=0$ for $i>1$. Consequently, the components of the asymptotic expansion (1.5) take the form

$$
\begin{aligned}
u_{k}^{\epsilon}(x) & =\hat{\varepsilon}_{k l} x_{l}+\varepsilon\left[\hat{\varepsilon}_{p q} \Xi_{k}^{p q}(y)-\hat{E}_{p} \Upsilon_{k}^{p}\right], \\
v^{\epsilon}(x) & =-\hat{E}_{l} x_{l}+\varepsilon\left[\hat{\varepsilon}_{p q} \Theta^{p q}(y)-\hat{E}_{p} \Pi^{p}\right] .
\end{aligned}
$$

Therefore, it is possible to obtain the components of the local strain field

$$
\varepsilon_{k l, x}\left(u^{\epsilon}\right)=\hat{\varepsilon}_{p q}\left[\delta_{k p} \delta_{l q}+\varepsilon_{k l, y}\left(\Xi^{p q}\right)\right]-\hat{E}_{p} \varepsilon_{k l, y}\left(\Upsilon^{p}\right),
$$

and the components of the local electric field intensity

$$
E_{l}\left(v^{\epsilon}\right)=\hat{E}_{p}\left[\delta_{p l}+\frac{\partial \Pi^{p}}{\partial y_{l}}\right]-\hat{\varepsilon}_{p q} \frac{\partial \Theta^{p q}}{\partial y_{l}},
$$

where

$$
E_{l}\left(v^{\epsilon}\right)=-\frac{\partial v^{\epsilon}}{\partial x_{l}}
$$

Note that the fields defined by (1.24) and (1.25) only depend on the local variable $y$.

\subsection{Application to a Binary Fibrous Piezocomposite with Perfect Contact Conditions at the Interfaces}

In this section, we apply the previously described method to a particular composite. We consider a two-phase fibrous composite consisting of identical circular cylinders embedded in a matrix. Both components are homogeneous piezoelectric materials. The axis of the fibers is parallel to the $x_{3}$-axis. The periodic distribution of the fibers follows a rectangular array as shown in Fig. 1.1. Perfect contact conditions on the interface $\Sigma^{\varepsilon}$ between the fibers and the matrix are assumed. The application of the above described homogenization process leads to 1.12$)-(1.20)$, with the addition of contact conditions on the interfaces.

The local problems (1.19)-(1.20) on the periodic cell $Y$ can be written as (BravoCastillero et al, 2001; Sabina et al, 2001):

- Problem $L^{p q}$ : To find the $Y$-periodic functions $\Xi_{k}^{p q}$ and $\Theta^{p q}$ such that:

$$
\begin{array}{ll}
\sigma_{i \delta, \delta}^{p q(\gamma)}=0, & \text { in } \quad Y_{\gamma}, \\
D_{\delta, \delta}^{p q(\gamma)}=0, & \text { in } \quad Y_{\gamma}, \\
\llbracket \Xi_{k}^{p q} \rrbracket=0, & \text { on } \quad \Sigma, \\
\llbracket \Theta^{p q} \rrbracket=0, & \text { on } \quad \Sigma,
\end{array}
$$



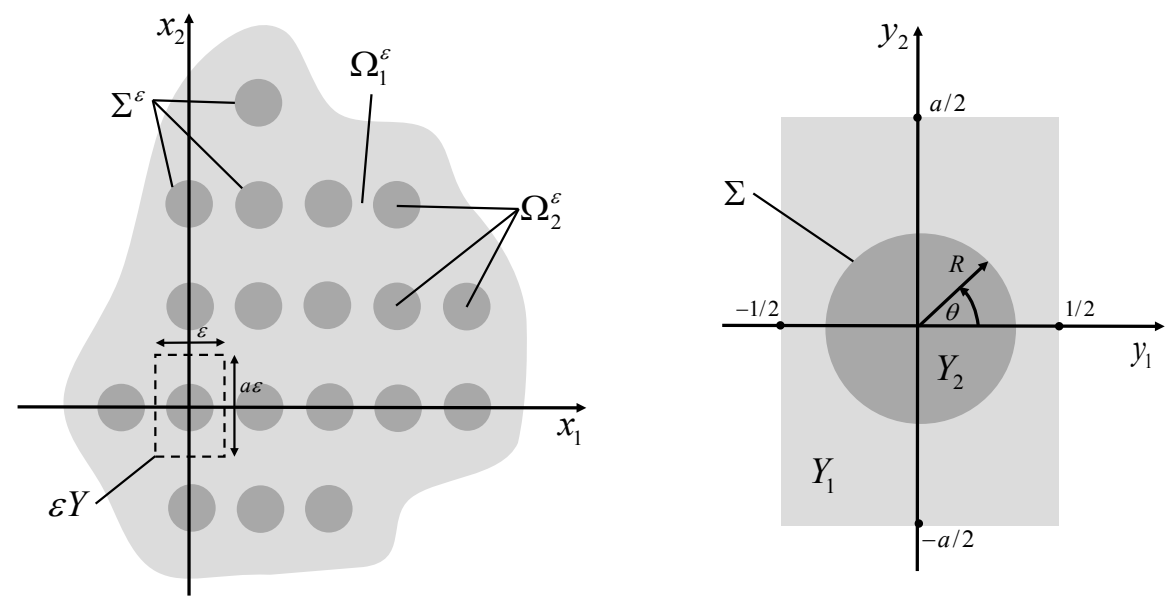

Fig. 1.1: Description of the cross-section of a two-phase fibrous periodic medium and the rectangular periodic cell

$$
\begin{aligned}
\llbracket \sigma_{i \delta}^{p q} n_{\delta} \rrbracket & =-\llbracket c_{i \delta p q} \rrbracket n_{\delta}, \quad \text { on } \quad \Sigma, \\
\llbracket D_{\delta}^{p q} n_{\delta} \rrbracket & =-\llbracket e_{\delta p q} \rrbracket n_{\delta}, \quad \text { on } \quad \Sigma, \\
\left\langle\Xi_{k}^{p q}\right\rangle & =0 \\
\left\langle\Theta^{p q}\right\rangle & =0
\end{aligned}
$$

with

$$
\begin{aligned}
& \sigma_{i \delta, \delta}^{p q(\gamma)}=c_{i \delta k \lambda}^{(\gamma)} \epsilon_{k \lambda, y}\left(\Xi^{p q(\gamma)}\right)+e_{\lambda i \delta}^{(\gamma)} \Theta_{, \lambda}^{p q(\gamma)} \\
& D_{\delta}^{p q(\gamma)}=e_{\delta k \lambda}^{(\gamma)} \epsilon_{k \lambda, y}\left(\Xi^{p q(\gamma)}\right)-\kappa_{\delta \lambda}^{(\gamma)} \Theta_{, \lambda}^{p q(\gamma)} .
\end{aligned}
$$

- Problem $L^{p}$ : Find the $Y$-periodic functions $\Upsilon_{k}^{p}$ and $\Pi^{p}$ such that:

$$
\begin{aligned}
\sigma_{i \delta, \delta}^{p(\gamma)} & =0, \quad \text { in } \quad Y_{\gamma}, \\
D_{\delta, \delta}^{p(\gamma)} & =0, \quad \text { in } \quad Y_{\gamma}, \\
\llbracket r_{k}^{p} \rrbracket & =0, \quad \text { on } \quad \Sigma, \\
\llbracket \Pi^{p} \rrbracket & =0, \quad \text { on } \quad \Sigma, \\
\llbracket \sigma_{i \delta, \delta}^{p} n_{\delta} \rrbracket & =-\llbracket e_{p i \delta} \rrbracket n_{\delta}, \quad \text { on } \quad \Sigma, \\
\llbracket D_{\delta}^{p} n_{\delta} \rrbracket & =-\llbracket \kappa_{\delta p} \rrbracket n_{\delta}, \quad \text { on } \quad \Sigma, \\
\left\langle\Upsilon_{k}^{p}\right\rangle & =0, \\
\left\langle\Pi^{p}\right\rangle & =0,
\end{aligned}
$$


with

$$
\begin{aligned}
& \sigma_{i \delta, \delta}^{p(\gamma)}=c_{i \delta k \lambda}^{(\gamma)} \epsilon_{k \lambda, y}\left(\Upsilon^{p(\gamma)}\right)+e_{\lambda i \delta}^{(\gamma)} \Pi_{, \lambda}^{p(\gamma)}, \\
& D_{\delta}^{p(\gamma)}=e_{\delta k \lambda}^{(\gamma)} \epsilon_{k \lambda, y}\left(\Upsilon^{p(\gamma)}\right)-\kappa_{\delta \lambda}^{(\gamma)} \Pi_{, \lambda}^{p(\gamma)},
\end{aligned}
$$

where $n_{\delta}$ are the components of the outer unit normal vector to the interface $\Sigma$, and $\llbracket \cdot \rrbracket=(\cdot)^{(1)}-(\cdot)^{(2)}$ denotes the contrast across $\Sigma$ taken from the matrix to the fiber. $\sigma_{i \delta}$ and $D_{\delta}$ are the components of local stress tensor and electric displacement vector, respectively. The Eqs. (1.26a) and (1.28a) are the corresponding equilibrium relations of solids bodies; (1.26b) and (1.28b) are the quasi-static approximation of Maxwell's equations in the absence of free charges. Perfect contact conditions on the interface are represented by $(1.26 \mathrm{c})-(1.26 \mathrm{f})$ and $(1.28 \mathrm{c})-(1.28 \mathrm{f})$. Conditions for uniqueness are given by $(1.26 \mathrm{~g})-(1.26 \mathrm{~h})$ and $(1.28 \mathrm{~g})-(1.28 \mathrm{~h})$. Formulae $(1.27 \mathrm{a})-(1.27 \mathrm{~b})$ and (1.29a) - 1.29b are the local constitutive relations. The Latin indices run from 1 to 3 , and the Greek ones from 1 to 2 . The comma denotes partial differentiation with respect to the local variable $y$.

\subsection{Local Problems for Fibrous Composites with Constituents of 622 Hexagonal Class}

In this work, we solve the local problems for the case corresponding to matrix and fibres made of piezoelectric materials with 622 hexagonal symmetry (Nye, 1957). These materials are characterized by eight independent constants $\left(k, m, l, n, p, s^{\prime}, t\right.$ and $u$ ), which are given by five elastic constants

$$
\begin{aligned}
& 2 k=c_{1111}+c_{1122}, \quad 2 m=c_{1111}-c_{1122}=c_{1212}, \\
& l=c_{1133}=c_{2233}, \quad n=c_{3333}, \quad p=c_{1313}=c_{2323}
\end{aligned}
$$

one piezoelectric constant

$$
s^{\prime}=-e_{123}=e_{213}=e_{231}=-e_{132}
$$

and two dielectric permittivity constants

$$
t=\kappa_{11}=\kappa_{22}, \quad u=\kappa_{33} .
$$

Consequently, the local problems $L^{11}, L^{22}, L^{33}$ and $L^{12}$ are exactly the same purely elastic problems which were solved in Nava-Gómez et al (2012) to obtain the effective coefficients $\underline{c}_{1111}, \underline{c}_{2211}, \underline{c}_{3311}, \underline{c}_{2222}, \underline{c}_{3322}, \underline{c}_{3333}$ and $\underline{c}_{1212}$. On the other hand, from $L^{3}$ one obtains that $\underline{\kappa}_{33}=\langle u\rangle$. Therefore, only four local problems $\left(L^{13}, L^{23}, L^{1}\right.$ and $L^{2}$ ) are relevant to obtain the remaining nonzero effective coefficients, which are $\underline{c}_{1313}, \underline{c}_{2323}, \underline{e}_{213}, \underline{e}_{123}, \underline{\kappa}_{11}$ and $\underline{\kappa}_{22}$.

Page: 9 job:editor macro:svmult.cls date/time:25-Jan-2018/8:32 
In the local problems $L^{13}, L^{23}, L^{1}$ and $L^{2}$, the relevant constitutive relations 1.27) and (1.29) can be summarized as

$$
\begin{aligned}
\sigma_{31} & =p \varepsilon_{13, y}-s^{\prime} E_{2}, \\
\sigma_{32} & =-s^{\prime} \varepsilon_{23, y}+t E_{1}, \\
D_{1} & =p \varepsilon_{23, y}+s^{\prime} E_{1}, \\
D_{2} & =s^{\prime} \varepsilon_{13, y}+t E_{2} .
\end{aligned}
$$

Thus, only three material properties are here involved: the longitudinal shear modulus $p$, the shear stress piezoelectric coefficient $s^{\prime}$ and the transverse permittivity constant $t$. The next subsections will be dedicated to the solution of these local problems and the further computation of the related effective coefficients.

\subsubsection{Local Problems $L^{23}$ and $L^{1}$}

These two problems can be stated in a unified form as follows

$$
\begin{aligned}
\Delta \Xi^{(\gamma)} & =0 \text { in } Y_{\gamma}, \\
\Delta \Theta^{(\gamma)} & =0 \text { in } Y_{\gamma}, \\
\llbracket \Xi \rrbracket & =0 \text { on } \Sigma, \\
\llbracket \Theta \rrbracket & =0 \text { on } \Sigma, \\
\llbracket\left(p \Xi_{, 1}-s^{\prime} \Theta_{, 2}\right) n_{1}+\left(p \Xi_{, 2}+s^{\prime} \Theta_{, 1}\right) n_{2} \rrbracket & =A n_{2} \text { on } \Sigma, \\
\llbracket\left(s^{\prime} \Xi_{, 2}-t \Theta_{, 1}\right) n_{1}-\left(s^{\prime} \Xi_{, 1}+t \Theta_{, 2}\right) n_{2} \rrbracket & =B n_{1} \text { on } \Sigma, \\
\langle\Xi\rangle & =0, \\
\langle\Theta\rangle & =0,
\end{aligned}
$$

where $\Delta$ is the two-dimensional Laplacian. Therefore, the solutions $\Xi^{(\gamma)}\left(\equiv \Xi_{3}^{23(\gamma)}\right)$ and $\Theta^{(\gamma)}\left(\equiv \Theta^{23(\gamma)}\right)$ are doubly periodic harmonic functions of the complex variable $z=y_{1}+i y_{2}$ defined in the rectangular cell $Y$ with periods $\omega_{1}=1$ and $\omega_{2}=a i$. The values of $A$ and $B$ in the right hand side of equations (1.34e) and (1.34f) for $L^{23}$ are $-\llbracket p \rrbracket$ and $-\llbracket s^{\prime} \rrbracket$ respectively. However, for the local problem $L^{1}$, these values are $A=-\llbracket s^{\prime} \rrbracket$ and $B=\llbracket t \rrbracket$, whereas $\Xi^{(\gamma)}\left(\equiv \Upsilon_{3}^{1(\gamma)}\right)$ and $\Theta^{(\gamma)}\left(\equiv \Pi^{1(\gamma)}\right)$.

The solution of [1.34) is sought as follows

$$
\begin{aligned}
& \Xi^{(1)}(z)=\mathfrak{I}\left\{-\frac{\delta_{2}}{\omega_{2}} a_{1} z+\sum_{k=1}^{\infty} a_{k} \frac{\zeta^{(k-1)}(z)}{(k-1) !}\right\}, \\
& \Theta^{(1)}(z)=\mathfrak{R}\left\{-\frac{\delta_{1}}{\omega_{1}} b_{1} z+\sum_{k=1}^{\infty} b_{k} \frac{\zeta^{(k-1)}(z)}{(k-1) !}\right\},
\end{aligned}
$$




$$
\begin{aligned}
& \Xi^{(2)}(z)=\mathfrak{I}\left\{\sum_{k=1}^{\infty} c_{k} z^{k}\right\}, \\
& \Theta^{(2)}(z)=\mathfrak{R}\left\{\sum_{k=1}^{\infty} d_{k} z^{k}\right\},
\end{aligned}
$$

where $a_{k}, b_{k}, c_{k}$ and $d_{k}$ are real and undetermined coefficients, $\Re\{z\}$ and $\mathfrak{I}\{z\}$ are, respectively, the real and imaginary part of the complex number $z$, and $\zeta(z)$ is the quasi-periodic Weierstrass Zeta function; whereas $\zeta^{(k)}(z)$ denotes the $k$-th derivative of periods $\omega_{1}$ and $\omega_{2}$. The superscript " $o$ " indicates that the summation is carried out only over the odd indices. $\Xi^{(\gamma)}$ is an even function of $\theta$, with $z=R e^{i \theta}$, and $\Theta^{(\gamma)}$ is an odd function of $\theta$.

The expressions for the undetermined constants $\delta_{\alpha}$ can be obtained from the quasi-periodicity of $\zeta(z)$

$$
\zeta\left(z+\omega_{\alpha}\right)-\zeta(z)=\delta_{\alpha}
$$

where

$$
\delta_{\alpha}=2 \zeta\left(\frac{\omega_{\alpha}}{2}\right)
$$

and Legendre's relation is fulfilled (see, for instance Lang, 1993).

The Laurent expansion about the origin for $\Xi^{(1)}$ and $\Theta^{(1)}$ can be written as

$$
\begin{aligned}
& \Xi^{(1)}(z)=\mathfrak{I}\left\{\sum_{l=1}^{\infty} a_{l} z^{-l}-\sum_{k=1}^{\infty} a_{k} \sum_{l=1}^{\infty} k \eta_{k l} z^{l}\right\}, \\
& \Theta^{(1)}(z)=\mathfrak{R}\left\{\sum_{l=1}^{\infty} b_{l} z^{-l}-\sum_{k=1}^{\infty} b_{k} \sum_{l=1}^{\infty} k \eta_{k l}^{\prime} z^{l}\right\},
\end{aligned}
$$

with

$$
\eta_{11}=\frac{\delta_{2}}{\omega_{2}}, \quad \eta_{11}^{\prime}=\frac{\delta_{1}}{\omega_{1}}, \quad \eta_{k l}=\eta_{k l}^{\prime}=\frac{(k+l-1) !}{k ! l !} S_{k+l} \quad \text { for } \quad k, l \neq 1,
$$

and the lattices sum $S_{k+l}$ is defined by

$$
S_{k+l}=\sum_{m, n}^{\prime}\left(m \omega_{1}+n \omega_{2}\right)^{-k-l}, \quad k+l \geq 3
$$

where the prime on the summation means that it excludes the term $m=n=0$. Now we use contact conditions $(1.34 \mathrm{c}-(1.34 \mathrm{f})$ to derive the following relations between the undetermined coefficients

$$
R^{l} c_{l}=-\left(R^{-l} a_{l}+\sum_{k=1}^{\infty} k \eta_{k l} R^{l} a_{k}\right)
$$




$$
\begin{aligned}
R^{l} d_{l} & =R^{-l} b_{l}-\sum_{k=1}^{\infty} k \eta_{k l}^{\prime} R^{l} b_{k}, \\
A R \delta_{1 l} & =\left(p^{(1)}+p^{(2)}\right) R^{-l} a_{l}-\llbracket p \rrbracket\left(\sum_{k=1}^{\infty} k \eta_{k l} R^{l} a_{k}\right)+\llbracket s^{\prime} \rrbracket\left(R^{-l} b_{l}-\sum_{k=1}^{\infty} k \eta_{k l}^{\prime} R^{l} b_{k}\right), \\
B R \delta_{1 l} & =\llbracket s^{\prime} \rrbracket\left(R^{-l} a_{l}+\sum_{k=1}^{\infty} k \eta_{k l} R^{l} a_{k}\right)-\left(t^{(1)}+t^{(2)}\right) R^{-l} b_{l}-\llbracket t \rrbracket\left(\sum_{k=1}^{\infty} k \eta_{k l}^{\prime} R^{l} b_{k}\right),
\end{aligned}
$$

for $l=1,3,5, \ldots$ Note that the coefficients $a_{k}$ and $b_{k}$ from $1.40 \mathrm{c}$ and $1.40 \mathrm{~d}$ are solutions of an infinite system of linear algebraic equations.

In order to solve the system (1.40), it is convenient to introduce the following change of variables

$$
\tilde{a}_{l}=\sqrt{l} R-l a_{l}, \quad \tilde{b}_{l}=\sqrt{l} R-l b_{l}, \quad \tilde{c}_{l}=\sqrt{l} R l c_{l}, \quad \tilde{d}_{l}=\sqrt{l} R l d_{l} .
$$

Thus, now we can write 1.40 as

$$
\begin{aligned}
(I+W) \mathfrak{V}_{1} & =\mathfrak{V}_{3}, \\
\left(I-W^{\prime}\right) \mathfrak{V}_{2} & =\mathfrak{V}_{4}, \\
\varphi_{11}^{(1)} \mathfrak{V}_{1}+\varphi_{12}^{(1)} \mathfrak{V}_{2}+\varphi_{11}^{(2)} W \mathfrak{V}_{1}+\varphi_{12}^{(2)} W^{\prime} \mathfrak{V}_{2} & =\widehat{V}_{1}, \\
\varphi_{21}^{(1)} \mathfrak{V}_{1}+\varphi_{22}^{(1)} \mathfrak{V}_{2}+\varphi_{21}^{(2)} W \mathfrak{V}_{1}+\varphi_{22}^{(2)} W^{\prime} \mathfrak{V}_{2} & =\widehat{V}_{2},
\end{aligned}
$$

where $I$ is the identity matrix, and the components $W$ and $W^{\prime}$ for $k=l=1$ are

$$
w_{11}=\frac{\delta_{2}}{\omega_{2}} R^{2}, \quad w_{11}^{\prime}=\frac{\delta_{1}}{\omega_{1}} R^{2},
$$

and, otherwise,

$$
w_{k l}=w_{k l}^{\prime}=\frac{(k+l-1) !}{(k-1) !(l-1) !} \frac{R^{k+l}}{\sqrt{k} \sqrt{l}} S_{k+l} .
$$

So, both $W$ and $W^{\prime}$ are real, symmetric and bounded; then we can use classical results from the theory of infinite systems (Kantorovich and Krylov, 1964). Furthermore,

$$
\begin{array}{ll}
\mathfrak{V}_{1}=\left(\tilde{a}_{1}, \tilde{a}_{3}, \tilde{a}_{5}, \ldots\right)^{T}, & \mathfrak{V}_{2}=\left(\tilde{b}_{1}, \tilde{b}_{3}, \tilde{b}_{5}, \ldots\right)^{T}, \\
\mathfrak{V}_{3}=\left(\tilde{c}_{1}, \tilde{c}_{3}, \tilde{c}_{5}, \ldots\right)^{T}, & \mathfrak{V}_{4}=\left(\tilde{d}_{1}, \tilde{d}_{3}, \tilde{d}_{5}, \ldots\right)^{T} .
\end{array}
$$

and all components of $\widehat{V}_{1}$ and $\widehat{V}_{2}$ are zero except the first ones, which are equal to $R \chi_{p}$ and $R \chi_{t}^{\prime}$, respectively, in $L^{23}$, and to $-R \chi_{p}$ and $-R \chi_{t}^{\prime}$ in $L^{1}$

$$
\chi_{p}=\frac{\llbracket p \rrbracket}{p^{(1)}+p^{(2)}}, \quad \quad \chi_{t}^{\prime}=\frac{\llbracket s^{\prime} \rrbracket}{t^{(1)}+t^{(2)}},
$$




$$
\chi_{p}^{\prime}=\frac{\llbracket s^{\prime} \rrbracket}{p^{(1)}+p^{(2)}}, \quad \chi_{t}=\frac{\llbracket t \rrbracket}{t^{(1)}+t^{(2)}} .
$$

Moreover, matrices $\Phi^{(\delta)}$, of components $\varphi_{\alpha \beta}^{(\delta)}$, are non-symmetric matrices and can be defined as

$$
\Phi^{(1)}=\left[\begin{array}{cc}
-1 & -\chi_{p}^{\prime} \\
-\chi_{t}^{\prime} & 1
\end{array}\right], \quad \Phi^{(2)}=\left[\begin{array}{cc}
\chi_{p} & \chi_{p}^{\prime} \\
-\chi_{t}^{\prime} & \chi_{t}
\end{array}\right] .
$$

Note that the knowledge of $\mathfrak{V}_{1}$ and $\mathfrak{V}_{2}$ is enough to solve the system (1.42). Equations 1.42 $\mathrm{c}$ and $1.42 \mathrm{~d}$ can be transformed into

$$
\begin{aligned}
\varphi_{11} \mathfrak{V}_{1}+\varphi_{12} \mathfrak{V}_{2}+W \mathfrak{V}_{1} & =V_{1}, \\
\varphi_{21} \mathfrak{V}_{1}+\varphi_{22} \mathfrak{V}_{2}+W^{\prime} \mathfrak{V}_{2} & =V_{2},
\end{aligned}
$$

or, in matrix form

$$
\Phi\left[\begin{array}{l}
\mathfrak{V}_{1} \\
\mathfrak{V}_{2}
\end{array}\right]+\left[\begin{array}{cc}
W & O \\
O & W^{\prime}
\end{array}\right]\left[\begin{array}{l}
\mathfrak{V}_{1} \\
\mathfrak{V}_{2}
\end{array}\right]=\left[\begin{array}{l}
V_{1} \\
V_{2}
\end{array}\right]
$$

where the $O$ denotes the null matrix and, in $L^{23}$, only the first component of $V_{1}$ is nonzero, and equal to $R$; and in $L^{1}$, only the first component of $V_{2}$ is nonzero, and equal to $-R$. Besides, we have

$$
\begin{aligned}
\Phi=\left[\Phi^{(2)}\right]^{-1} \Phi^{(1)} & =\frac{1}{\Lambda}\left[\begin{array}{cc}
-\chi_{t}+\chi_{p}^{\prime} \chi_{t}^{\prime} & -\chi_{p}^{\prime}\left(1+\chi_{t}\right) \\
-\chi_{t}^{\prime}\left(1+\chi_{p}\right) & \chi_{p}-\chi_{p}^{\prime} \chi_{t}^{\prime}
\end{array}\right], \\
\Lambda & =\chi_{p} \chi_{t}+\chi_{p}^{\prime} \chi_{t}^{\prime},
\end{aligned}
$$

In order to solve the infinite system (1.50) it must be truncated as follows

$$
H \mathfrak{V}=V,
$$

where $\mathfrak{V}=\left(\mathfrak{V}_{1 i}, \mathfrak{V}_{2 i}\right)^{T}$ and $V=\left(V_{1 i}, V_{2 i}\right)^{T}$, for $i=1,3, \ldots, 2 n_{0}-1$. The natural number $n_{0}$ denotes the truncation order.

The general form of the components of the principal matrix $H=\left(h_{i j}\right)$ of 1.53 can be defined as follows:

$$
h_{i j}=\left\{\begin{array}{l}
\text { for } i \text { odd }\left\{\begin{array}{l}
h_{i i}=\varphi_{11}+w_{i i} \\
h_{i j}=w_{i j}, \quad \text { if } j \text { odd } \\
h_{i i+1}=\varphi_{12}
\end{array}\right. \\
\text { for } i \text { even }\left\{\begin{array}{l}
h_{i i}=\varphi_{22}+w_{i-1 i-1}^{\prime} \\
h_{i j}=w_{i-1 j-1}^{\prime}, \quad \text { if } j \text { even } \\
h_{i i-1}=\varphi_{21},
\end{array}\right.
\end{array}\right.
$$


From system (1.53) the values of $\tilde{a}_{i}$ and $\tilde{b}_{i}$ can be obtained using the inverse matrix method to solve systems; and then the values of $a_{i}$ and $b_{i}$ by reversing the change of variables described in (1.41), resulting in the following formulae

$$
a_{i}=R \mathscr{H}_{i} U, \quad b_{i}=R \mathscr{H}_{i+1} U,
$$

where $\mathscr{H}_{i}$ is the $i$-th row of $H^{-1}$, which is the inverse matrix of $H$.

\subsubsection{Effective Coefficients Related with the Local Problems $L^{23}$ and $L^{1}$}

The nonzero effective coefficients which can be computed from the local problems $L^{23}$ and $L^{1}$ are

$$
\begin{aligned}
\underline{c}_{2323} & =p_{v}+\left\langle p \Xi_{3,2}^{23}-s^{\prime} \Theta_{, 1}^{23}\right\rangle, \\
-\underline{e}_{123} & =s_{v}^{\prime}+\left\langle s^{\prime} \Xi_{3,2}^{23}+t \Theta_{, 1}^{23}\right\rangle=s_{v}^{\prime}+\left\langle p \Upsilon_{3,2}^{1}+s^{\prime} \Pi_{, 1}^{1}\right\rangle, \\
\underline{\kappa}_{11} & =t_{v}+\left\langle t \Pi_{, 1}^{1}-s^{\prime} \Upsilon_{3,2}^{1}\right\rangle,
\end{aligned}
$$

where $p_{v}=c_{1} p^{(1)}+c_{2} p^{(2)}$, with $c_{1}+c_{2}=1$ and $c_{2}=\pi R^{2} / a$.

After that, the application of Green's theorem, the doubly periodicity of the local functions and the conditions on $\Sigma$ leads to the following expressions (see, for instance, Sabina et al, 2001; Aguiar et al, 2013)

$$
\begin{aligned}
\underline{c}_{2323} & =p^{(1)}\left(1+\frac{2 \pi}{a} a_{1}^{23}\right), \\
-\underline{e}_{123} & =s^{\prime(1)}\left(1+\frac{2 \pi t^{(1)}}{a s^{\prime(1)}} b_{1}^{23}\right)=s^{\prime(1)}\left(1+\frac{2 \pi p^{(1)}}{a s^{(1)}} a_{1}^{1}\right), \\
\underline{\kappa}_{11} & =t^{(1)}\left(1-\frac{2 \pi}{a} b_{1}^{1}\right),
\end{aligned}
$$

where only the residues $a_{1}$ and $b_{1}$, of $\Xi^{(1)}$ and $\Theta^{(1)}$, are relevant for computing such effective coefficients. The superindices on $a_{1}$ and $b_{1}$ indicate the local problem which is solved in order to use the formulas (1.55) for $i=1$.

\subsubsection{Local Problems $L^{13}$ and $L^{2}$ and Related Effective Coefficients}

The study of the local problems $L^{13}$ and $L^{2}$ and the related effective coefficients is very similar as above. Then only the main results will be summarized in this subsection. 
The simultaneous formulation of these problems consists in to find the doubly periodic functions $\Xi^{(\gamma)}$ and $\Theta^{(\gamma)}$ such that:

$$
\begin{aligned}
\Delta \Xi^{(\gamma)} & =0 \text { in } Y_{\gamma}, \\
\Delta \Theta^{(\gamma)} & =0 \text { in } Y_{\gamma}, \\
\llbracket \Xi \rrbracket & =0 \text { on } \Sigma, \\
\llbracket \Theta \rrbracket & =0 \text { on } \Sigma, \\
\llbracket\left(p \Xi_{, 1}-s^{\prime} \Theta_{, 2}\right) n_{1}+\left(p \Xi_{2}+s^{\prime} \Theta_{, 1}\right) n_{2} \rrbracket & =C n_{1} \text { on } \Sigma, \\
\llbracket\left(s^{\prime} \Xi_{, 2}-t \Theta_{, 1}\right) n_{1}-\left(s^{\prime} \Xi_{, 1}+t \Theta_{, 2}\right) n_{2} \rrbracket & =D n_{2} \text { on } \Sigma, \\
\langle\Xi\rangle & =0, \\
\langle\Theta\rangle & =0 .
\end{aligned}
$$

The solutions $\Xi^{(\gamma)}\left(\equiv \Xi_{3}^{13(\gamma)}\right)$ and $\Theta^{(\gamma)}\left(\equiv \Theta^{13(\gamma)}\right)$ are $Y$-periodic harmonic functions depending on $z=y_{1}+i y_{2}$ with periods $\omega_{1}=1$ and $\omega_{2}=a i$. The values of $C$ and $D$ in the right hand side of equations (1.62e) and (1.62f) for $L^{13}$ are $-\llbracket p \rrbracket$ and $\llbracket s^{\prime} \rrbracket$, respectively. For the local problem $L^{1}$, these values are $C=\llbracket s^{\prime} \rrbracket$ and $D=\llbracket t \rrbracket$, whereas $\Xi^{(\gamma)}\left(\equiv \Upsilon_{3}^{2(\gamma)}\right)$ and $\Theta^{(\gamma)}\left(\equiv \Pi^{2(\gamma)}\right)$.

According to the interface conditions $1.62 \mathrm{e}$ ) and $(1.62 \mathrm{f}]$, the solution of 1.62 is sought as

$$
\begin{aligned}
& \Xi^{(1)}(z)=\mathfrak{R}\left\{-\frac{\delta_{2}}{\omega_{2}} a_{1} z+\sum_{k=1}^{\infty} a_{k} \frac{\zeta^{(k-1)}(z)}{(k-1) !}\right\}, \\
& \Theta^{(1)}(z)=\mathfrak{I}\left\{-\frac{\delta_{1}}{\omega_{1}} b_{1} z+\sum_{k=1}^{\infty} b_{k} \frac{\zeta^{(k-1)}(z)}{(k-1) !}\right\}, \\
& \Xi^{(2)}(z)=\mathfrak{R}\left\{\sum_{k=1}^{\infty} c_{k} z^{k}\right\}, \\
& \Theta^{(2)}(z)=\mathfrak{I}\left\{\sum_{k=1}^{\infty} d_{k} z^{k}\right\} .
\end{aligned}
$$

Furthermore, using similar ideas to those discussed previously, the following formulae for the related effective coefficients can be obtained

$$
\begin{aligned}
\underline{c}_{1313} & =p^{(1)}\left(1-\frac{2 \pi}{a} a_{1}^{13}\right), \\
\underline{e}_{213} & =-s^{\prime(1)}\left(1+\frac{2 \pi t^{(1)}}{a s^{(1)}} b_{1}^{13}\right)=s^{\prime(1)}\left(1+\frac{2 \pi p^{(1)}}{a s^{(1)}} a_{1}^{2}\right), \\
\underline{\kappa}_{22} & =t^{(1)}\left(1+\frac{2 \pi}{a} b_{1}^{2}\right),
\end{aligned}
$$


where $a_{1}$ and $b_{1}$ are also the residues of $\Xi^{(1)}$ and $\Theta^{(1)}$.

Now, by using the change of variable (1.41) it is possible to arrive to the infinite system to compute $\tilde{a}_{1}$ and $\tilde{b}_{1}$ :

$$
\begin{aligned}
(I-M) \mathfrak{V}_{1} & =\mathfrak{V}_{3}, \\
\left(I+M^{\prime}\right) \mathfrak{V}_{2} & =-\mathfrak{V}_{4}, \\
\psi_{11}^{(1)} \mathfrak{V}_{1}+\psi_{12}^{(1)} \mathfrak{V}_{2}+\psi_{11}^{(2)} M \mathfrak{V}_{1}+\psi_{12}^{(2)} M^{\prime} \mathfrak{V}_{2} & =\widehat{V}_{1}, \\
\psi_{21}^{(1)} \mathfrak{V}_{1}+\psi_{22}^{(1)} \mathfrak{V}_{2}+\psi_{21}^{(2)} M \mathfrak{V}_{1}+\psi_{22}^{(2)} M^{\prime} \mathfrak{V}_{2} & =\widehat{V}_{2},
\end{aligned}
$$

where the only difference to system $(1.42)$ is that

$$
m_{11}=\frac{\delta_{1}}{\omega_{1}} R^{2}, \quad m_{11}^{\prime}=\frac{\delta_{2}}{\omega_{2}} R^{2},
$$

and, otherwise,

$$
m_{k l}=m_{k l}^{\prime}=\frac{(k+l-1) !}{(k-1) !(l-1) !} \frac{R^{k+l}}{\sqrt{k} \sqrt{l}} S_{k+l} .
$$

Besides,

$$
\Psi^{(1)}=\left[\begin{array}{cc}
1 & -\chi_{p}^{\prime} \\
-\chi_{t}^{\prime} & -1
\end{array}\right], \quad \Psi^{(2)}=\left[\begin{array}{cc}
\chi_{p} & -\chi_{p}^{\prime} \\
\chi_{t}^{\prime} & \chi_{t}
\end{array}\right],
$$

and, as above, we find the matrix $\Psi$, which is given by

$$
\Psi=\left[\Psi^{(2)}\right]^{-1} \Psi^{(1)}=\frac{1}{\Lambda}\left[\begin{array}{cc}
\chi_{t}-\chi_{p}^{\prime} \chi_{t}^{\prime} & -\chi_{p}^{\prime}\left(1+\chi_{t}\right) \\
-\chi_{t}^{\prime}\left(1+\chi_{p}\right) & -\chi_{p}+\chi_{p}^{\prime} \chi_{t}^{\prime}
\end{array}\right]
$$

Therefore, we can write the system in the following way

$$
\Psi\left[\begin{array}{l}
\mathfrak{V}_{1} \\
\mathfrak{V}_{2}
\end{array}\right]+\left[\begin{array}{cc}
M & O \\
O & M^{\prime}
\end{array}\right]\left[\begin{array}{l}
\mathfrak{V}_{1} \\
\mathfrak{V}_{2}
\end{array}\right]=\left[\begin{array}{l}
V_{1} \\
V_{2}
\end{array}\right],
$$

where only the first components of $V_{1}$ and $V_{2}$ can be different from zero, and equal to $R$. In the case of problem $L^{13}$, the first component of $V_{1}$ is the one that is nonzero; in the case of problem $L^{2}$, only the first component of $V_{2}$ is equal to $R$.

System (1.72) can be written in the form (1.53) with the particularity that the components of the principal matrix $H=\left(h_{i j}\right)$ are defined by

$$
h_{i j}=\left\{\begin{array}{l}
\text { for } i \text { odd }\left\{\begin{array}{l}
h_{i i}=\psi_{11}+m_{i i}, \\
h_{i j}=m_{i j}, \quad \text { if } j \text { odd }, \\
h_{i i+1}=\psi_{12},
\end{array}\right. \\
\text { for } i \text { even }\left\{\begin{array}{l}
h_{i i}=\psi_{22}+m_{i-1 i-1}^{\prime}, \\
h_{i j}=m_{i-1 j-1}^{\prime}, \quad \text { if } j \text { even, } \\
h_{i i-1}=\psi_{21},
\end{array}\right.
\end{array}\right.
$$


Finally, the corresponding values of $a_{1}$ and $b_{1}$ can be computed by using Eqs. 1.55, respectively.

\subsubsection{On the Computation of the Local Fields from the Solutions of the Local Problem $L^{13}$}

As we can note, the analytical solutions of the local problems are expressed in terms of the local coordinates $\rho$ and $\theta$. However, to study the behavior of the local fields (1.24) and 1.25 it is necessary to compute their derivatives with respect to the Cartesian coordinates $y_{\delta}$, and then the chain's rule must be used

$$
\frac{\partial}{\partial y_{1}}=\cos \theta \frac{\partial}{\partial \rho}-\frac{\sin \theta}{\rho} \frac{\partial}{\partial \theta}, \quad \frac{\partial}{\partial y_{2}}=\sin \theta \frac{\partial}{\partial \rho}+\frac{\cos \theta}{\rho} \frac{\partial}{\partial \theta} .
$$

For instance, the solutions $\Xi^{(\gamma)}\left(\equiv \Xi_{3}^{13(\gamma)}\right)$ and $\Theta^{(\gamma)}\left(\equiv \Theta^{13(\gamma)}\right)$, of the local problem $L^{13}$, can be written as

$$
\begin{aligned}
& \Xi^{(1)}(\rho, \theta)=\sum_{l=1}^{2 n_{0}-1} a_{l} \rho^{-l} \cos (l \theta)-\sum_{k=1}^{2 n_{0}-1} a_{k} \sum_{l=1}^{2 n_{0}-1} k \eta_{k l} \rho^{l} \cos (l \theta), \\
& \Theta^{(1)}(\rho, \theta)=-\sum_{l=1}^{2 n_{0}-1} b_{l} \rho^{-l} \sin (l \theta)-\sum_{k=1}^{2 n_{0}-1} b_{k} \sum_{l=1}^{2 n_{0}-1} k \eta_{k l}^{\prime} \rho^{l} \sin (l \theta), \\
& \Xi^{(2)}(\rho, \theta)=\sum_{k=1}^{2 n_{0}-1} c_{k} \rho^{k} \cos (k \theta), \\
& \Theta^{(2)}(\rho, \theta)=\sum_{k=1}^{2 n_{0}-1} d_{k} \rho^{k} \sin (k \theta),
\end{aligned}
$$

where the constants $a_{l}, b_{l}, c_{l}$ and $d_{l}\left(l=1,3, \ldots, 2 n_{0}-1\right)$ can be obtained from the solution of 1.67a - 1.67d for a truncation order $n_{0}$.

As an example, we consider the following homogeneous boundary conditions: $\hat{\varepsilon}_{13}=1$ and $\hat{\varepsilon}_{p q}=0$, for the strain tensor components, and $\hat{E}_{p}=0$ for the components of electric field intensity vector. Then, Eqs. (1.24) and (1.25) take the form

$$
\varepsilon_{k l}(y)=\delta_{k 1} \delta_{l 3}+\delta_{k 3} \delta_{l 1}+2 \varepsilon_{k l, y}\left(\Xi^{13}\right), \quad E_{l}(y)=-2 \frac{\partial \Theta^{13}}{\partial y_{l}} .
$$

Finally, it is possible to compute the components of the local fields from the solutions of the local problem $L^{13}$ as follows 


$$
\begin{aligned}
\varepsilon_{13}(y) & =1+\frac{\partial \Xi_{3}^{13}}{\partial y_{1}}, & \varepsilon_{23}(y) & =\frac{\partial \Xi_{3}^{13}}{\partial y_{2}} \\
E_{1}(y) & =-2 \frac{\partial \Theta^{13}}{\partial y_{1}}, & E_{2}(y) & =-2 \frac{\partial \Theta^{13}}{\partial y_{2}} .
\end{aligned}
$$

which can be computed by using the expressions $(1.75)$ and the chain rule. A similar procedure allows to obtain analytical expressions for the local fields related with the solutions of the local problems $L^{23}, L^{1}$ and $L^{2}$.

\subsection{Numerical Examples}

Some examples are presented, which include comparisons between the analytical results derived from the present model (PM) and those obtained with the fast Fourier transform (FFT) numerical method (Moulinec and Suquet, 1998, Michel et al, 2001; Brenner, 2009, 2010). For the case of a fiber-reinforced matrix, we have considered the data previously used in López-López et al (2005). They correspond to a collagen matrix with collagen-hydroxyapatite (HA) fibers. For the case of a porous piezoelectric matrix, we have considered the data used in Aguiar et al (2013) corresponding to bone material (Table 1.1).

In Figs. 1.2 1.5, the evolution of the effective coefficients with the fiber volume fraction $c_{2}$ is shown. The range of variation of $c_{2}$ goes from zero up to the percolation limit when two neighboring fibers or holes get in contact. The results are normalized by the properties of the matrix. In Fig. [1.2, a square periodic cell $(a=1)$ is considered and the results from López-López et al (2005) are reproduced. In Figs. 1.4 and 1.5, a rectangular periodic cell (for $a=2$ ) is discussed. Finally, in Figs. [1.6 and 1.7, the spatial distribution of the local fields is illustrated.

Table 1.1: Piezoelectric properties used for the computations (López-López et al 2005: Aguiar et al, 2013). $\epsilon_{0}$ denotes the permittivity of free-space.

\begin{tabular}{lcc} 
& Collagen & HA \\
\hline Longitudinal shear modulus $p(\mathrm{GPa})$ & 1.400 & 2.697 \\
Shear strain piezoelectric coefficient $d=s^{\prime} / p(\mathrm{pC} / \mathrm{N})$ & 0.062 & 0.041 \\
Transverse permittivity constant $t / \epsilon_{0}($ no units) & 2.825 & 2.509 \\
\hline & Bone \\
\hline Longitudinal shear modulus $p(\mathrm{GPa})$ & 8.2 \\
Shear stress piezoelectric coefficient $s^{\prime}(\mathrm{N} / \mathrm{Vm})$ & $2.214 \times 10^{-3}$ \\
Transverse permittivity constant $t / \epsilon_{0}$ (no units) & 6.85 \\
\hline
\end{tabular}

Page: 18 job:editor macro: svmult.cls date/time:25-Jan-2018/8:32 


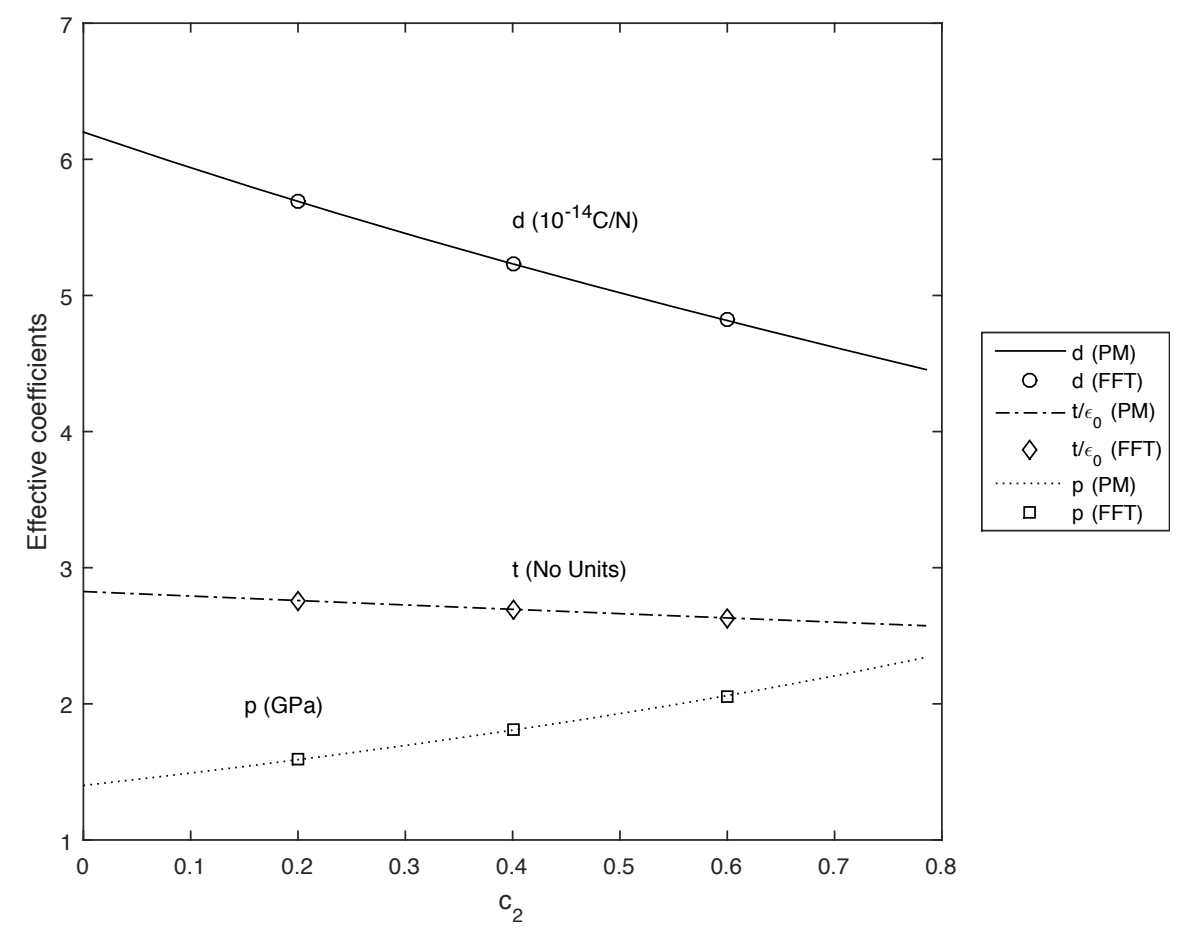

Fig. 1.2: The elastic, piezoelectric and dielectric effective coefficients for a twophase fibrous piezocomposite with a square $(a=1)$ periodic distribution of fibres. Comparisons of the results obtained with the present model (PM) with those derived by the FFT

\subsubsection{Square Array Distribution}

In Fig. 1.2, numerical results are shown for a fiber reinforced material with square periodic cell $(a=1)$. In this case the following equalities were numerically verified: $\underline{c}_{1313}=\underline{c}_{2323}, \underline{\kappa}_{11}=\underline{\kappa}_{22}$ and $\underline{e}_{213}=-\underline{e}_{123}$. Then, for simplicity, $p, t$ and $s^{\prime}$ are used to denote the effective elastic, dielectric permittivity and piezoelectric coefficients, respectively. Also, $d=s^{\prime} / p$ denotes the effective shear strain piezoelectric coefficient. A truncation order $n_{0}=2$ is used for the computations with the present model.

A good agreement can be observed between the semi-analytical (PM) and the numerical results (FFT). The curves corresponding to the present model reproduce those published in López-López et al (2005).

The results shown in Fig. 1.3 correspond to a porous material and, as in Fig. 1.2 the periodic cell is square. Here, the equalities $\underline{c}_{1313}=\underline{c}_{2323}=\underline{\kappa}_{11}=\underline{\kappa}_{22}$ and $\underline{e}_{213}=-\underline{e}_{123}$ stand and were numerically verified. A good agreement between the $\mathrm{PM}$ results and those from the numerical FFT method can be observed whatever the 


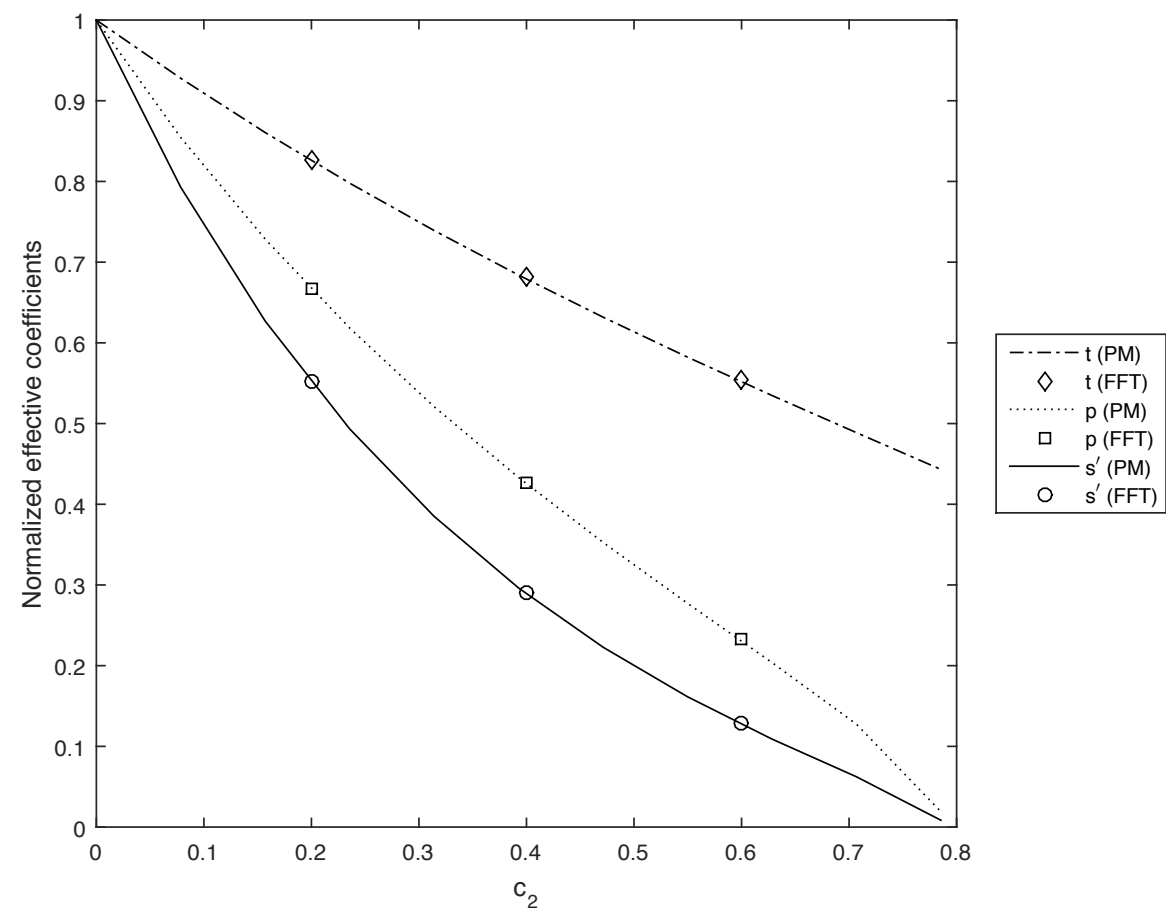

Fig. 1.3: The normalized elastic, piezoelectric and dielectric effective coefficients for a piezocomposite with a square $(a=1)$ periodic distribution of cylindrical holes. Comparisons between the results derived from the present model (PM) with those derived from the Fast Fourier Transform numerical method (FFT)

fiber volume fraction. The results shown for the present model were obtained using a truncation order $n_{0}=5$.

\subsubsection{Rectangular Array Distribution}

In Figs. 1.4 and 1.5. piezoelectric composites with a rectangular periodic distribution of the fibers are examined for $a=2$. In Fig. 1.4. a two-phase composite with an orthotropic effective behavior is studied. In Fig. 1.5. a porous piezocomposite which preserves the symmetry properties of the matrix is considered. In both figures, an excellent agreement can be observed, for all the range of fiber area fractions, among the analytical (PM) and numerical (FFT) results and for all the normalized effective coefficients. Note that both models capture the expected global behavior for each type of composite. The truncation order used for the computations with the present model was $n_{0}=4$. 

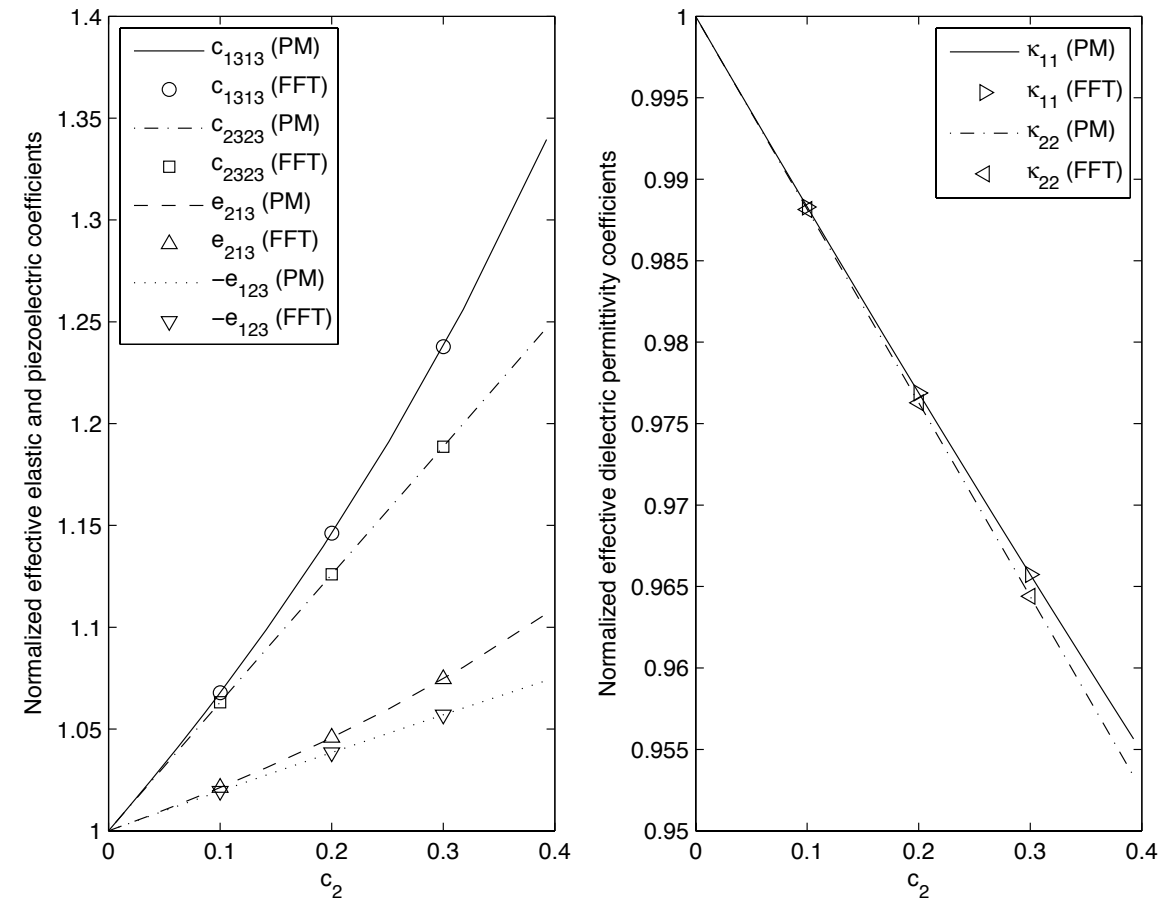

Fig. 1.4: The normalized elastic, piezoelectric and dielectric effective coefficients for a two-phase fibrous piezocomposite with a rectangular $(a=2)$ periodic distribution of fibres. Comparisons of the results derived from the present model (PM) with those derived by the Fast Fourier Transform numerical method (FFT)

\subsubsection{Spatial Distribution of Local Fields}

The local fields defined by (1.24) and (1.25) depend on the solution of all local problems. However, for specific homogeneous boundary conditions only one local problem needs to be solved in order to compute the local fields. For instance, as was shown above, the solutions of the local problem $L^{13}$ are enough to compute the local fields given by (1.77)-(1.78). In Figs. 1.6 and 1.7, the distribution of the components $\epsilon_{13}$ and $E_{1}$, for a truncation order $n_{0}=7$ is plotted. In both cases the radius of the fiber is $R=0.35$. A significant variation is predicted in the matrix at the fiber-matrix interface normal to the $x_{1}$ and $x_{2}$ axis respectively, and almost uniform fields are predicted within the fibers. The latter is an expected result. Let us consider, for example, the Eq. 1.77): using Eqs. (1.75c) and (1.74) it yields

$$
\varepsilon_{13}(y)=1+\frac{\partial \Xi_{3}^{13}}{\partial y_{1}}, \quad \varepsilon_{13}(y)=1+\sum_{k=1}^{2 n_{0}-1} k c_{k} \rho^{k-1} \cos (k-1) \theta .
$$



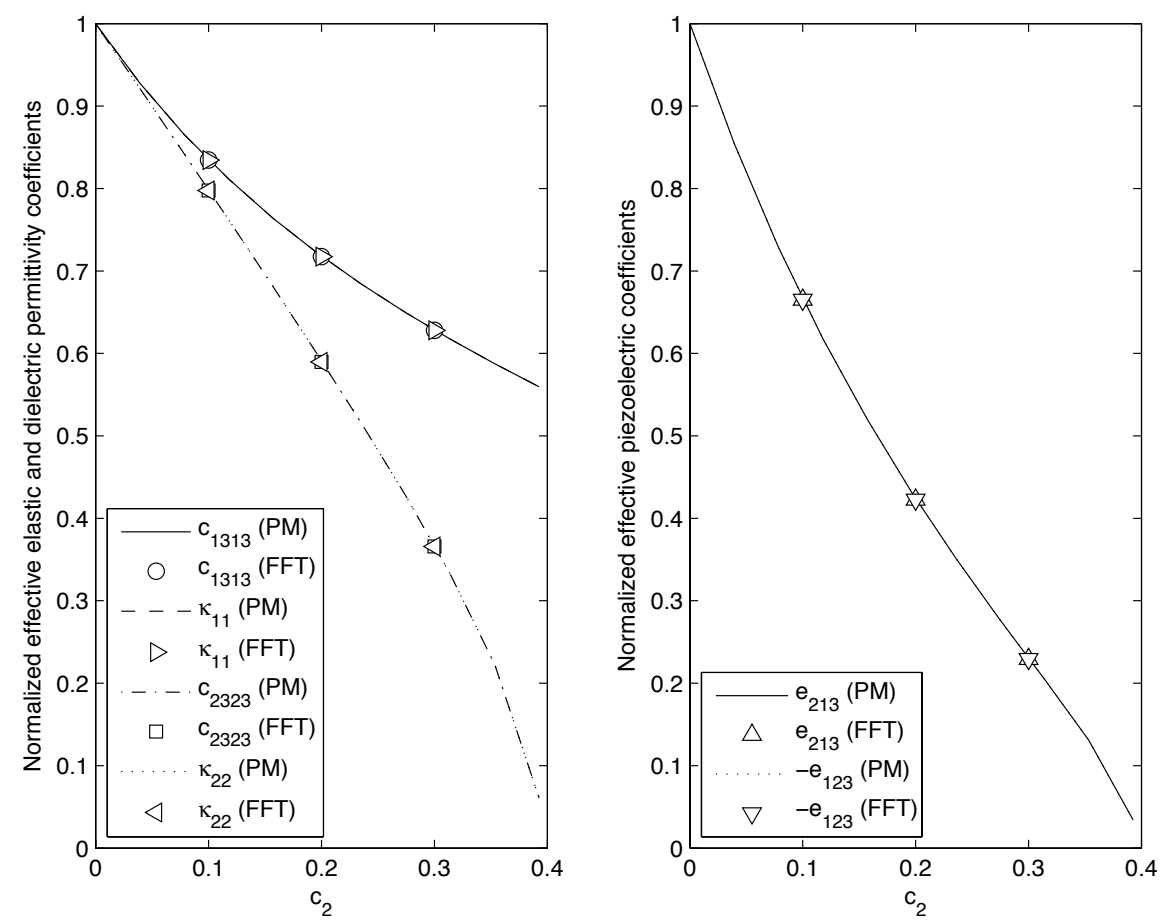

Fig. 1.5: The normalized elastic, piezoelectric and dielectric effective coefficients for a piezocomposite with a rectangular $(a=2)$ periodic distribution of cylindrical holes. The results derived from the present model (PM) and those derived from the Fast Fourier Transform numerical method (FFT) are compared

The sum in the right hand side of Eq. 1.79$]_{2}$ can be easily majored by the sum

$$
\left|c_{1}\right| \sum_{k=1}^{2 n_{0}-1} k\left(\frac{1}{2}\right)^{k-1} .
$$

Hence, the aforementioned sum should have little to nil influence in the final value of the field inside the fiber, since the value of $c_{1}$ for the given data set is very small.

The results shown in Fig. 1.6 were obtained using the formula (1.77) corresponding to the solutions of the local problem $L^{13}$, for homogeneous boundary conditions of the type $\hat{\epsilon}_{p q}=\delta_{1 p} \delta_{3 q}$ and $\hat{E}_{p}=0$. This field is similar to that predicted via FFT, as can be seen in Fig. 1.6, where the relative error between the computation using Eq. 1.77) and the one using FFT is plotted, i.e. $|\mathrm{PM}-\mathrm{FFT}| / \mathrm{PM}$. It can be seen that in the matrix both methods coincide, while in the fiber they predict behaviors with about a 20\% difference. This difference can be explained by the little influence of the sum in the right hand side of Eq. [1.79] in the actual value of the field inside the fiber. 




(a)



(b)

Fig. 1.6: (a) Component $\epsilon_{13}$ of the local strain field within the fiber composite with square arrangement for an axial shear strain load $\hat{\epsilon}_{13}=\hat{\epsilon}_{31}=1$; (b) Absolute error between the computation in (a) using the present model (1.77) and the FFT numerical scheme 
The variation shown in Fig. [1.7 was computed for homogeneous boundary conditions of the type $\hat{\epsilon}_{p q}=0$ and $\hat{E}_{p}=\delta_{1 p}$. Such conditions transform 1.24) and 1.25 into

$$
\begin{aligned}
& \varepsilon_{13}(y)=-\frac{\partial \gamma_{3}^{1}}{\partial y_{1}}, \quad \varepsilon_{23}(y)=-\frac{\partial \gamma_{3}^{1}}{\partial y_{2}} \\
& E_{1}(y)=1+\frac{\partial \Pi^{1}}{\partial y_{1}}, \quad E_{2}(y)=\frac{\partial \Pi^{1}}{\partial y_{2}} .
\end{aligned}
$$

Consequently, only the solutions of the problem $L^{1}$ are involved in 1.81 . Notice that again in Fig. 1.7 the relative error of the computations through the present method and FFT was plotted and they show coincidence in the matrix, while the differences inside the fiber are not greater than a $6 \%$.

\subsection{Concluding Remarks}

Analytical formulae for the effective coefficients were obtained for binary fibrous composites with 622 hexagonal piezoelectric components and a rectangular distribution of the unidirectional circular fibers. These results contain as particular cases those reported in López-López et al (2005) and Aguiar et al (2013) where only the square periodic cell was considered. Analytical expressions to study the fluctuations of the components of the local strain and the local electric field intensity are explicitly given. For the binary and the porous piezoelectric materials, the present model has been successfully compared to the results obtained with the FFT numerical homogenization method (Brenner, 2009, 2010).

Acknowledgements Support provided by the French Priority Solidarity Fund (Campus France FSP Cuba 29896TD), the Brazilian Coordination for the Improvement of Higher Education Personnel (Project CAPES 88881.030424/2013-01), and the CoNaCyT 129658 is gratefully acknowledged. The authors are also grateful to Ana Pérez Arteaga and Ramiro Chávez for computational support. JB acknowledges the Cátedra Extraordinaria IIMAS and PREI-DGAPA, UNAM.

\section{References}

Aguiar AR, Bravo-Castillero J, Rodríguez-Ramos R, da Silva UP (2013) Effective electromechanical properties of 622 piezoelectric medium with unidirectional cylindrical holes. J App Mech 80(5):050,905

Alfonso-Rodríguez R, Bravo-Castillero J, D PL (2017) Local fields in two-phase fibrous piezocomposites with 622 constituents. Math Methods App Sci 40:3221-3229

Bakhvalov N, Panasenko GP (1989) Homogenisation: Averaging Processes in Periodic Media. Kluwer, Oxford

Berger H, Gabbert U, Köppe H, Rodríguez-Ramos R, Bravo-Castillero J, Guinovart-Díaz R, Otero JA, Maugin GA (2003) Finite element and asymptotic homogenization methods applied to smart composite materials. Comp Mech 33:61-67 


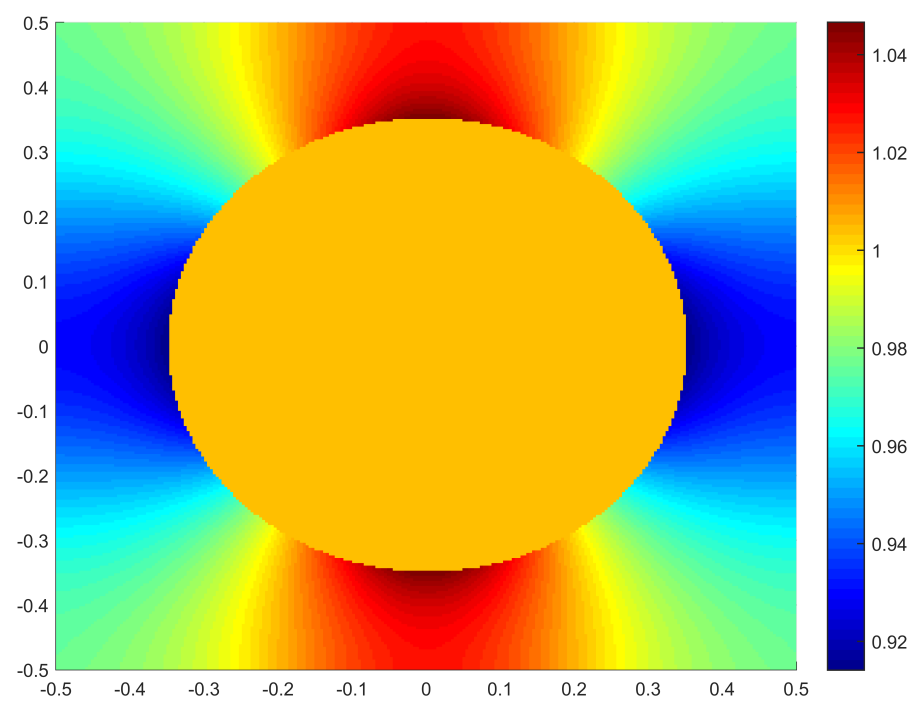

(a)

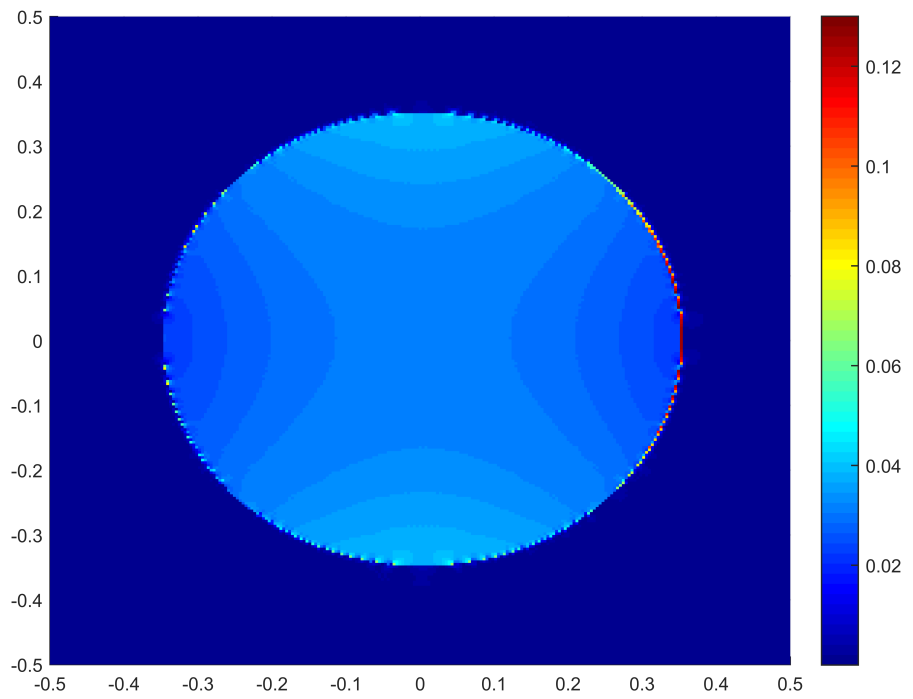

(b)

Fig. 1.7: (a) Component $E_{1}$ of the local electric field intensity within the fiber composite with square arrangement for an unitary uniaxial applied electric field $\hat{E}_{1}=1$; (b) Absolute error between the computation in (a) using the present model (1.82) and FFT

Page:25 job:editor macro:svmult.cls date/time:25-Jan-2018/8:32 
Berger H, Kari S, Gabbert U, Rodríguez-Ramos R, Bravo-Castillero J, Guinovart-Díaz R, Sabina FJ, Maugin GA (2006) Unit cell models of piezoelectric fiber composites for numerical and analytical calculation of effective properties. J Smart Mat Struct 15:451-458

Bravo-Castillero J, Guinovart-Díaz R, Otero JA, Rodríguez-Ramos R (1997) Electromechanical properties of continuous fibre-reinforced piezoelectric composites. Mech Comp Mater 33:670680

Bravo-Castillero J, Otero JA, Rodríguez-Ramos R, Bourgeat A (1998) Asymptotic homogenization of laminated piezocomposite materials. Int J Solids Struct 35:527-541

Bravo-Castillero J, Guinovart-Díaz R, Sabina FJ, Rodríguez-Ramos R (2001) Closed-form expressions for the effective coefficients of fibre-reinforced composite with transversely isotropic constituents - II. Piezoelectric and square symmetry. Mech Mater 33:237-248

Brenner R (2009) Numerical computation of the response of piezoelectric composites using Fourier transform. Phys Rev B 79:184,106

Brenner R (2010) Computational approach for composite materials with coupled constitutive laws. Smart Mater Struct 61:919-927

Fukada E (1984) Piezoelectricity of natural biomaterials. Ferroelec 60:285-296

Galka A, Telega JJ, Wojnar R (1992) Homogenization and thermopiezoelectricity. Mech Res Comm 19:315-324

Galka A, Telega JJ, Wojnar R (1996) Some computational aspects of homogenization of thermopiezoelectric composites. Computer Assisted Mechanics and Engineering Sciences 3:133-154

Kantorovich LV, Krylov VI (1964) Approximate Methods of Higher Analysis. Wiley, New York

Lang S (1993) Complex Analysis, 3rd edn. Graduate Texts in Mathematics, Springer, New York

López-López E, Sabina F, Bravo-Castillero J, Guinovart-Díaz R, Rodríguez-Ramos R (2005) Overall electromechanical properties of a binary composite with 622 symmetry constituents. Antiplane shear piezoelectric state. Int J Solids Struct 42:5765-5777

Maugin GA, Turbé N (1991) Homogenization of piezoelectric composites via Bloch expansion. Int J Appl Electromagnetics in Mat 2:135-140

Michel JC, Moulinec H, Suquet P (2001) A computational scheme for linear and non-linear composites with arbitrary phase contrast. Int J Numer Meth Engng 52:139-160

Moulinec H, Suquet P (1998) A numerical method for computing the overall response of nonlinear composites with complex microstructure. Comput Methods Appl Mech Engrg 157:69-94

Nava-Gómez GG, Camacho-Montes HF, Sabina FJ, Rodríguez-Ramos R, Fuentes L, Guinovart-Díaz $\mathrm{R}$ (2012) Elastic properties of an orthotropic binary fiber-reinforced composite with auxetic and conventional constituents. Mech Mater 48:1-25

Nye JF (1957) Physical Properties of Crystals: Their Representation by Tensors and Matrices. Clarendon Press, Oxford

Otero JA, Bravo-Castillero J, Guinovart-Díaz R, Rodríguez-Ramos R, Maugin GA (2003) Analytical expressions of effective constants for a piezoelectric composite reinforced with square crosssection fibers. Arch Mech 55:357-371

Rodríguez-Ramos R, Otero JA, Bravo-Castillero J, Sabina FJ (1996) Electromechanical properties of laminated piezoelectric composites. Mech Comp Mater 32:410-417

Sabina FJ, Rodríguez-Ramos R, Bravo-Castillero J, Guinovart-Díaz R (2001) Closed-form expressions for the effective coefficients of fibre-reinforced composite with transversely isotropic constituents - II. Piezoelectric and hexagonal symmetry. J Mech Phys Solids 49:1463-1479

Sevostianov I, da Silva UP, Aguiar AR (2014) Green's function for piezoelectric 622 hexagonal crystals. Int J Eng Sci 84:18-28

Sixto-Camacho LM, Bravo-Castillero J, Brenner R, Guinovart-Díaz R, Mechkour H, RodriguezRamos R, Sabina FJ (2013) Asymptotic homogenization of periodic thermo-magneto-electroelastic heterogeneous media. Comp Math App 66:2056-2074

Telega JJ (1991) Piezoelectricity and homogenization. application to biomechanics. In: Maugin GA (ed) Continuum Models and Discrete Systems, Longman, Essex, vol 2, pp 220-229

Turbé N, Maugin GA (1991) On the linear piezoelectricity of composite materials. Math Meth Appl Sci 14:403-412

Page:26 job:editor macro:svmult.cls date/time:25-Jan-2018/8:32 